\title{
Alimentación y estilos de vida saludables percibidos en estudiantes universitarios del área de la salud, de tres licenciaturas
}

\author{
Botello-Márquez Mariela*, Villanueva-Lugo Norma Edith**, Ruiz-Vargas Nancy Viridiana***, \\ Gallegos-Torres Ruth Magdalena****
}

\section{Resumen}

- Introducción: La calidad de la alimentación en jóvenes universi-

- tarios juega un papel importante para el desempeño académi-

- co y calidad de vida y salud en la vida adulta. Objetivo: Determi-

- nar la alimentación y estilos de vida percibidos en estudiantes

- universitarios del área de la salud, de tres licenciaturas de una

- institución pública. Metodología: Estudio transversal, descriptivo.

- Se encuestaron alumnos de primer semestre. Se aplicó Cues-

- tionario de Hábitos Alimentarios (Alfa de Cronbach de 0.82).

- Proyecto aprobado por un Comité de Investigación y Bioética.

- Resultados: I 58 alumnos de las tres licenciaturas: 32\% hombres,

- $68 \%$ mujeres. Edad promedio de 20.3 años \pm 2.4 .54\% de los

- alumnos de Enfermería consideró alimentarse sanamente con

- alguna frecuencia, 55\% de Fisioterapia y 57\% de Educación

- Física. Más del 50\% de los participantes consideran tener una

- dieta de regular a buena, resaltando que a veces tienen una

- alimentación saludable o bien cuidan la calidad de los alimentos

- o el contenido calórico de los alimentos; de este último punto,

- los alumnos de Educación Física son los que menos lo realizan

- (30\%) y los de Fisioterapia más (64\%). Conclusiones: Múltiples

- autores evidencian el cambio en los estilos de vida de los estu-

- diantes universitarios al ingresar a la universidad, por lo que es

- relevante identificar patrones alimenticios de los estudiantes al

- ingreso y durante la carrera universitaria, así como factores que

- pueden afectar o beneficiar dichos patrones, para establecer

- medidas de mantenimiento o corrección de la alimentación, con

- la finalidad de tener estudiantes sanos y que cuenten con con-

- ductas saludables que favorezcan una adultez sana. LUXMÉDICA,

- AÑO 13, NÚMERO 39, S EPT-DIC 2018, PP 55-62.

- Palabras clave: evaluación nutricional, estudiantes del área de la salud, conductas saludables.

\section{Abstract}

Introduction: The quality of nutrition in university students plays an important role in academic performance and quality of life and health in adult life. Objective:To determine the diet and lifestyles perceived in health sciences university students from three bachelor's degrees from a public institution. Methods: Cross-sectional, descriptive study; first semester students were surveyed. The Food Habits Questionnaire (Cronbach's alpha of 0.82) was applied. The project was approved by a Research and Bioethics Committee. Results: One hundred fifty-eight students from three bachelor's degrees were included, 32\% men, $68 \%$ women, with an average age of 20.3 years \pm 2.4 . 54\% of the nursing students considered they eat healthy with some frequency, 55\% of physical therapy students, and 57\% of physical education. More than $50 \%$ of the participants consider having a regular to good diet, highlighting that sometimes they have a healthy diet or they consider the quality of the food or the caloric content of the food; of this last point, physical education students are the ones who care the least (30\%) and physical therapy students the most (64\%). Conclusions: Multiple authors show the change in the lifestyles of university students when they enter university, therefore, it is important to identify the students' eating patterns on admission and during university, as well as factors that may affect or benefit these patterns, to establish measures of maintenance or correction of food intake, in order to have healthy students and have healthy behaviors that promote a healthy adulthood. LUX MÉDICA, AÑO 13, NÚMERO 39, S EPT-DIC 2018, PP 55-62.

Keywords: nutritional evaluation, health sciences students, healthy behaviors

Pasante del Servicio Social, Licenciatura en Enfermería. Facultad de Enfermería. Universidad Autónoma de Querétaro. Correo electrónico: maguis-_@hotmail.com Pasante del Servicio Social, Licenciatura en Enfermería. Facultad de Enfermería. Universidad Autónoma de Querétaro. Correo electrónico: villanuevalugo.edith@ gmail.com

** Licenciada en Enfermería, estudiante de la Maestría en Enfermería. Facultad de Enfermería. Universidad Autónoma de Querétaro. Correo electrónico: nancy.ruiz@ uaq.mx

*** Doctora en Ciencias de la Salud. Docente de tiempo Completo. Facultad de Enfermería, Universidad Autónoma de Querétaro.

Fecha de recibido: 10 de marzo 2018

Fecha de aceptación: 4 de junio 2018

Correspondencia: Dra Ruth Magdalena Gallegos Torres. Facultad de Enfermería. Universidad Autónoma de Querétaro. Cerro de las Campanas sin número. Colonia Las Campanas, código postal 76010. Santiago de Querétaro, Querétaro. Teléfono 01442192 1200, móvil 4421446592, correo electrónico: isisrmgx@gmail.com 


\section{Introducción}

La Organización Mundial de la Salud ${ }^{1}$ señala que el estilo de vida se refiere a una forma general de vida basada en la interacción entre las condiciones de vida en un sentido amplio y los patrones individuales de conducta determinados por factores socioculturales y características personales.

Los estilos de vida abarcan una serie de dimensiones tales como la actividad física, alimentación, relaciones sociales, aspectos psicoemocionales y espirituales ${ }^{2}$. En este sentido, el desarrollo de un estilo de vida saludable está vinculado con aspectos, además de la alimentación, como reducción de estrés, enfermedades, emociones negativas, ejercicio, calidad del sueño, administración del tiempo, entre otros ${ }^{3}$.

Entre los elementos que constituyen el estilo de vida alimenticio se han considerado las conductas y preferencias relacionadas con el tipo, horario y cantidad de alimentación ${ }^{4}$. En este sentido, la dieta y la nutrición constituyen un estilo de vida muy importante para promover y mantener la buena salud a lo largo de toda la vida ${ }^{5}$.

Al respecto, se reconoce que "mantener un estilo de vida saludable es especialmente importante entre quienes fomentan y promocionan la salud en la comunidad como los estudiantes universitarios del área de ciencias de la salud", ya que las decisiones que tomen repercutirán a mediano y largo plazo².

En México, los nuevos estilos de vida han traído consigo hábitos no saludables que aumentan el riesgo de desarrollar enfermedades crónico-degenerativas. En lo que respecta a la alimentación, se está viviendo una transición, pues se ha pasado de una dieta rica en verduras, frutas, cereales y leguminosas a una dieta rica en grasas y azucares refinados. Además se suma que se lleva un estilo de vida sedentario, que suele vincularse con otros comportamientos perjudiciales para la salud como el consumo regular de tabaco y alcohol. Todo ello trae como consecuencia el sobrepeso y la obesidad ${ }^{4}$.

Al respecto, existen estudios que revelan que los estudiantes de Ciencias de la Salud no aplican en sus hábitos nutricionales los conocimientos que están adquiriendo en sus estudios universitarios, manteniendo ingestas desequilibradas de macronutrientes $\mathrm{e}$ importantes déficits nutricionales de micronutrientes ${ }^{6}$.

Sin embargo, el estado nutricional de los universitarios puede verse afectado por las presiones a que están sometidos debido a largas jornadas de estudio, cambios de horarios, tensiones psicológicas y a que sus hábitos alimentarios muchas veces son inadecuados ${ }^{7}$. Por este motivo, es que el grupo de estudiantes universitarios se considera vulnerable, ya que además de lo anterior, se agrega "escasez de tiempo y dinero, tradiciones, creencias y valores, los medios de comunicación y publicidad, el ideal de delgadez, los alimentos disponibles, estados de ánimo" ${ }^{8}$, entre otros.

El resultado de este proceso tiene injerencia directa y principal en el rendimiento académico del estudiante, el cual estará enton- 
ces mediado por las características del estilo de vida recientemente adoptado para enfrentar al entorno y sus condiciones establecidas ${ }^{9}$.

Como parte del ambiente universitario y de las responsabilidades que directivos y docentes deben de tener con el recurso humano, que son los alumnos, es necesario no solo participar de los procesos de ciencia, cultura y arte, sino el de promover estilos de vida saludables en los estudiantes ${ }^{10}$.

En este sentido, es importante considerar la alimentación que manejan los estudiantes, sobre todo el alcance de la comida "chatarra", es decir, alimentos inadecuados, poco nutritivos, de los cuales, un consumo de más de dos veces por semana podría llegar a ocasionar "lesiones y enfermedades en el organismo dado que contiene sustancias tóxicas como conservadores, saborizantes, colorantes, estabilizantes, alto contenido calórico, grasas trans, están hechos a base de harina y un excesivo contenido de sal o azúcar" ${ }^{11}$.

Lo anterior abre una brecha respecto a espacios saludables, los cuales son necesarios para beneficiar a la comunidad, ya que la universidad se convierte en un segundo hogar para los estudiantes, puesto que allí se concentra gran parte del tiempo del joven, en la búsqueda de una profesión que conduzca a mejorar el estilo de vida en general ${ }^{12}$.

Sin embargo, la modificación de conductas y actitudes que generen una cultura saludable no depende únicamente de las personas, sino también de la estructura organizacional, políticas y decisiones estratégicas que se adopten desde los cargos directivos de las universidades ${ }^{10}$.

Los estilos de vida saludables son importantes en el desarrollo humano, ya que por medio de estos se puede lograr un crecimiento positivo en el ámbito personal. Generar autocuidado es parte fundamental para tener una buena salud y por tanto una vida con bienestar $^{8}$.

La educación, la información y la comunicación sobre un estilo de vida saludable, juegan un papel muy importante para la salud 9 . Al respecto, en Colombia se realizó un estudio sobre la prevalencia de sobrepeso, obesidad, comportamientos alimentarios y de actividad física en estudiantes universitarios con 204 estudiantes de la Facultad de Ciencias de la Salud; y respecto a los hábitos de alimentación se menciona que los principales factores de riesgo asociados a sobrepeso y obesidad fueron consumir menos de tres comidas al día, no desayunar todos los días y no consumir regularmente frutas ${ }^{13}$.

Es con base en lo anterior que este estudio tuvo como objetivo determinar la alimentación y estilos de vida percibidos en estudiantes universitarios del área de la salud, de tres licenciaturas de una institución pública del estado de Querétaro, considerando la Licenciatura en Enfermería, Licenciatura en Fisioterapia y Licenciatura en Educación Física y Ciencias del Deporte. 


\section{Material y métodos}

Se trató de un estudio descriptivo transversal, llevado a cabo en estudiantes de la Universidad Autónoma de Querétaro, Facultad de Enfermería inscritos en las 3 Licenciaturas: Enfermería, Fisioterapia, Educación Física y Ciencias del Deporte, donde el universo es el $100 \%$ de los alumnos del 1 er semestre, por lo que se integra una muestra de 158 estudiantes.

Se trabajó con los estudiantes que se encontraban presentes durante la aplicación del cuestionario, bajo el consentimiento informado para participar en el estudio. El tipo de muestreo fue por conveniencia, dado que al ser una cantidad accesible de estudiantes, se procuró que todos los que aceptaron participar contestaran el cuestionario. Para el acercamiento a los estudiantes, se visitaron los campus en una sola ocasión.

El cuestionario elaborado por Pilar Castro Rodríguez, Diego Bellido Guerrero y Sonia Pertega Díaz ${ }^{14}$ se denomina "Cuestionario de hábitos alimentarios" el cual cuenta con ocho factores, integrados en 36 preguntas. Los factores son los siguientes: I, aspectos sobre el consumo de azúcar en la dieta; II, cumplimiento de normas dietéticas relacionadas con una alimentación saludable; III, práctica habitual o sistemática de ejercicio físico; IV, preguntas relativas al hecho de ingerir alimentos por bienes- tar psicológico; $\mathrm{V}$, contenido calórico; VI, tipo de alimentos que consumen frecuentemente; VII, conocimiento y control de los sujetos sobre qué y cuánto comen; VIII, preguntas relacionadas con el consumo de bebidas alcohólicas. Las preguntas del cuestionario poseen opciones múltiples para las posibles respuestas, las cuales consideraban frecuencia de consumo. Las opciones de respuesta fueron nunca, pocas veces, alguna frecuencia, muchas veces y siempre, teniendo valores del 1 al 5 según el tipo de pregunta y el factor al que se hacía referencia.

El cuestionario fue piloteado en 15 estudiantes de la Licenciatura en Enfermería de la Universidad Autónoma de Querétaro, Campus San Juan del Rio, trabajando correcciones mínimas de formato más no de contenido. El acercamiento con la comunidad estudiantil se llevó a cabo en julio del 2017.

Para el procesamiento de los datos de la recolección definitiva, se realizó una base de datos en el programa SPSS v.20. Para el análisis se utilizó estadística descriptiva manejando promedios, además de obtener las medidas de tendencia central (media, mediana, moda y desviación estándar).

Dentro de los aspectos éticos se siguieron los principios establecidos en el Reglamento de la Ley General de Salud en Materia de Investigación para la Salud ${ }^{15}$.

\section{| | | | | | | | | | | | | | | | | | | | | | | | | | | | | | | | | | | | | | | | | | | | | | | | | | | | | | | | | | | | | | | | | | | | | | | | | | | | | | | | | | | | | | | | | | | | | | | | | | | | | | | | ||}

\section{Resultados}

Se trabajó con 72 alumnos de la Licenciatura en Enfermería, 56 de la Licenciatura en Fisioterapia y 30 de la Licenciatura en Educación Física y Ciencias del Deporte. Del total, $68 \%$ fueron mujeres. A continuación se muestran los resultados por licenciatura.

\section{Licenciatura en Enfermería}

$54 \%$ se alimentan sanamente; $61 \%$ practica con alguna frecuencia actividad física; en el bienestar psicológico pocas de las veces se encuentran estables con un $60 \%$. Con alguna frecuencia, el $57 \%$ consume un alto contenido calórico. 58\% tiene conocimiento y control sobre el tema (gráfica 1).

\section{Licenciatura en Fisioterapia}

$55 \%$ refirió mantener con alguna frecuencia, una alimentación saludable. $66 \%$ realiza ejercicio físico. Sobre el bienestar psicológico, $75 \%$ se identifican estables mentalmente. Con alguna frecuencia, el $64 \%$ consume un alto contenido calórico. El $66 \%$ cuenta con conocimiento y control del tema (gráfica 2). 


\section{Licenciatura en Educación Física y Ciencias del Deporte}

$57 \%$ de los participantes refirió mantener una alimentación saludable con alguna frecuencia. Realizan ejercicio físico el $63 \%$ con alguna frecuencia. En bienestar psicológico, $63 \%$ lo presenta con alguna frecuencia;
$30 \%$ consume un bajo contenido calórico con alguna frecuencia y $63 \%$ tiene conocimiento y control del tema (gráfica 3).

En la gráfica 4 se muestran resultados sobre la percepción de los alumnos de las tres licenciaturas, sobre la calidad de su dieta.

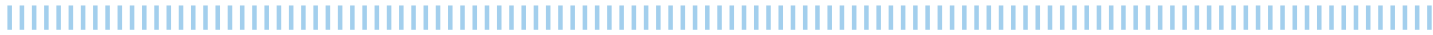

\section{Discusión}

La estancia del estudiante en la universidad debe ser un escenario saludable que potencialice un estilo de vida saludable físico, psicológico y social. Los universitarios están inmersos en diferentes cambios sociales como estrés, alcoholismo o alguna otra adicción, enfermedades no transmisibles y conductas alimentarias de riesgo que son producto de una conducta previa, lo que contribuye en su estilo de vida.

Al respecto, los resultados de esta investigación mostraron que en promedio, $55 \%$ de los estudiantes de las tres carreras refiere que mantiene una alimentación saludable, lo cual es bajo comparado con los hallazgos de Fernández ${ }^{16}$, quien trabajó con 119 estudiantes de la Carrera de Psicología Clínica del Área de la Salud Humana de la Universidad Nacional de Loja, donde encontró que el $74.78 \%$ tienen una alimentación adecuada. Sin embargo, los datos coinciden con el estudio de Jiménez, quienes trabajaron con 197 alumnos de la Universidad Autónoma de Yucatán, de los cuales, $56.36 \%$ indicó un manejo regular de su alimentación. De Piero ${ }^{17}$, señala que en el estudio de 175 estudiantes universitarios, se encontró que estos, en general, presentaron una alimentación desequilibrada, monótona e insuficiente en cantidad y calidad. Durán, de la misma manera, señala que en 1418 estudiantes universitarios estudiados, hay una malnutrición por exceso, por un alto consumo de alimentos no saludables ${ }^{18}$.

En relación a la práctica de actividad física, al momento de ingresar a la universi- dad, $63 \%$ la realizaba con alguna frecuencia. Estos resultados son superiores a los hallazgos de Saliba ${ }^{8}$, quien trabajó con 114 estudiantes de diversas carreras del área de la salud, evidenciando que la práctica de ejercicios físicos no era frecuente, realizándolo el $32.5 \%$. Sin embargo, en el estudio de Tamayo ${ }^{11}$ los porcentajes (en 100 alumnos) fueron ligeramente menores, donde $59 \%$ sí realiza actividad física. Por sexo, Chávez ${ }^{19}$ encontró que las mujeres dedican menos tiempo a esta actividad.

Los resultados encontrados en este estudio, aunque generales, evidencian que es un reto cuidar la salud del estudiante universitario. Sobre todo en el caso de los alumnos encuestados, ya que se encontraban por iniciar el primer semestre de sus respectivas carreras. En este sentido, se reconoce la necesidad de realizar estudios de seguimiento, de manera tal que se aprecie el cambio en los estilos de vida y alimentación de los jóvenes, a lo largo de la carrera.

Los hallazgos siguen reforzando que en las instituciones educativas es necesario tener un trabajo más detallado y preciso con los alumnos, al orientarles en su alimentación, estilos de vida y en aquellos factores que los estén alterando o influyendo y que además impactan en su rendimiento académico.

Por ello, frente a la figura del estudiante universitario, directivos, docentes y personal que forma parte del ambiente académico, debe reforzar su trabajo en el cuidado de la salud, reflexión sobre conductas sanas y en cómo esto repercutirá en la vida futura, sobre todo en la salud. 


\section{Conclusiones}

Los resultados permiten contar con un punto de reflexión sobre las áreas del conocimiento que es necesario trabajar con los alumnos que se forman en salud, ya que estos, de manera directa o indirecta, en un futuro, tendrán la responsabilidad de guiar individuos o grupos, hacia un estilo de vida saludable por lo que también, de parte de las autoridades y docentes formadores, se tiene la responsabilidad de garantizar un estilo de vida sano, llevado de la mano de información adecuada.

\section{Gráfica I}

\section{Resultados del cuestionario de hábitos alimenticios en estudiantes de la Licenciatura en Enfermería}

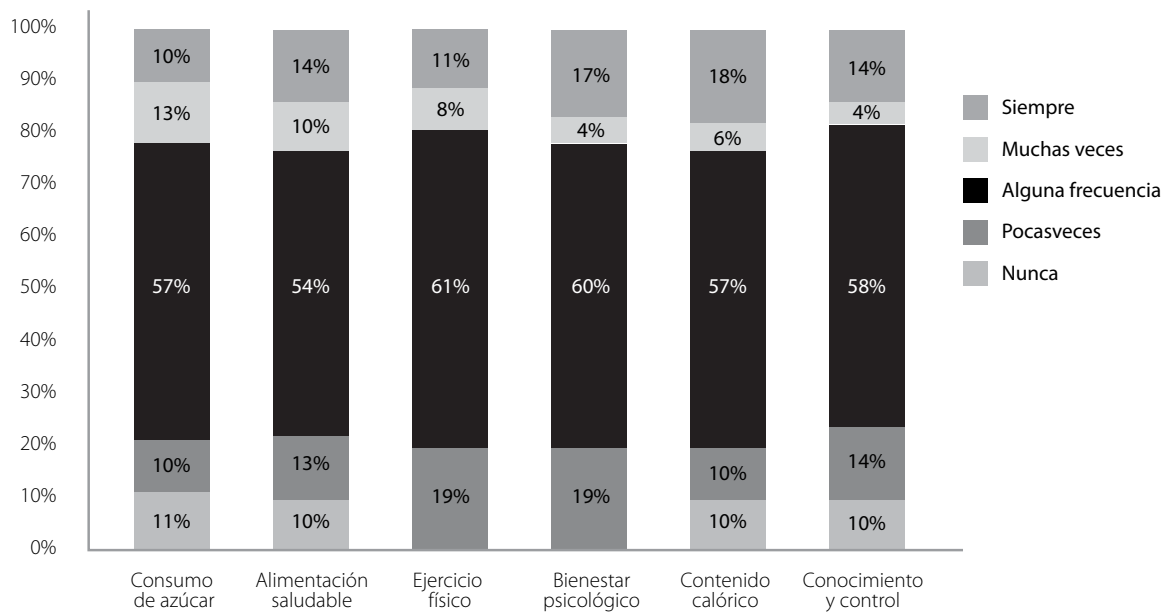

Fuente: Cuestionario de hábitos alimentarios de Castro, Bellido y Pertega, 2010.

\section{Gráfica 2}

Resultados del cuestionario de hábitos alimenticios en estudiantes de la Licenciatura en Fisioterapia

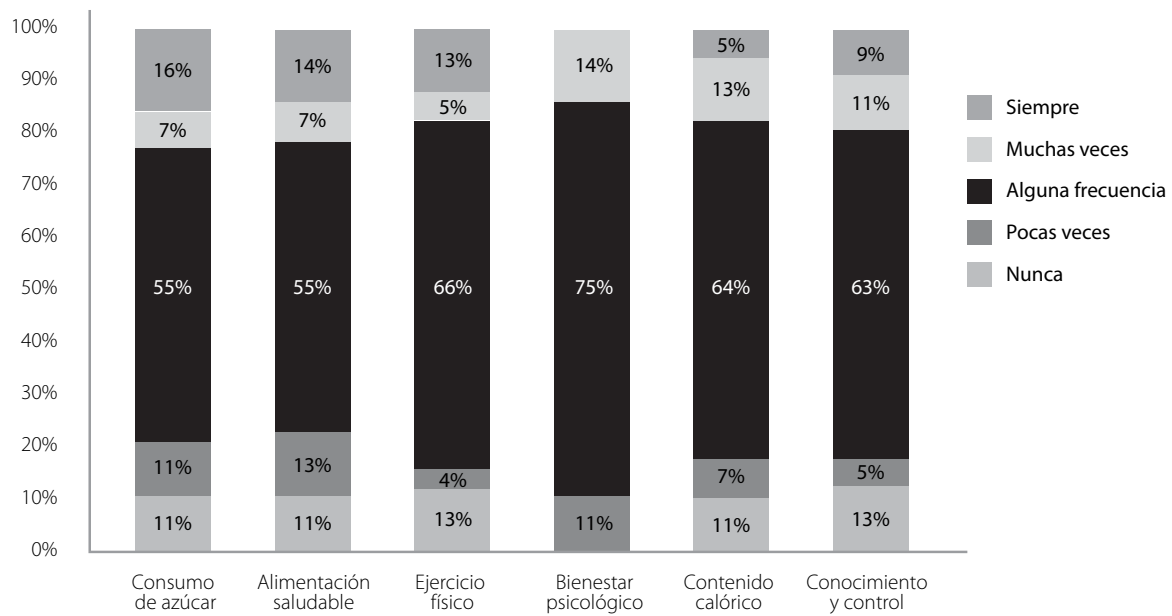

Fuente: Cuestionario de hábitos alimentarios de Castro, Bellido y Pertega, 2010. 


\section{Gráfica 3}

Resultados del cuestionario de hábitos alimenticios en estudiantes de la Licenciatura en Educación Física y Ciencias del Deporte

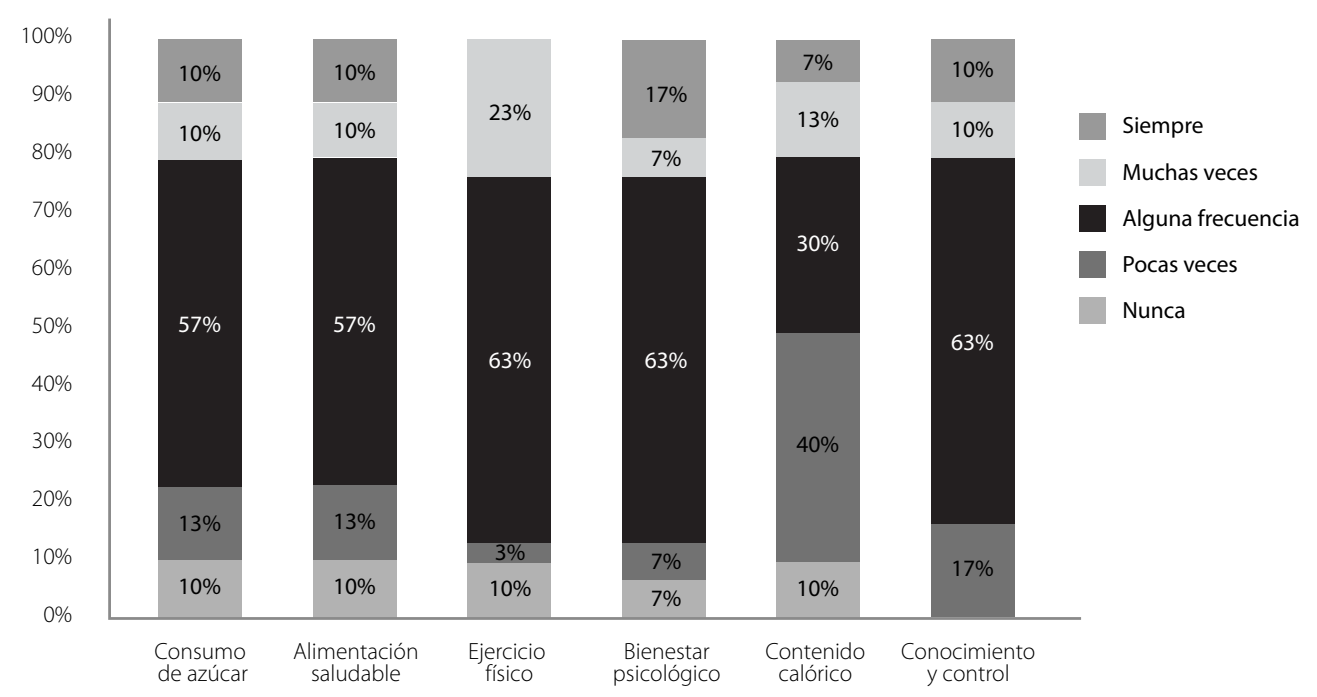

Fuente: Cuestionario de hábitos alimentarios de Castro, Bellido y Pertega, 2010.

\section{Gráfica 4}

Resultados de la pregunta "creo que mi dieta es..." en los alumnos de las tres licenciaturas.

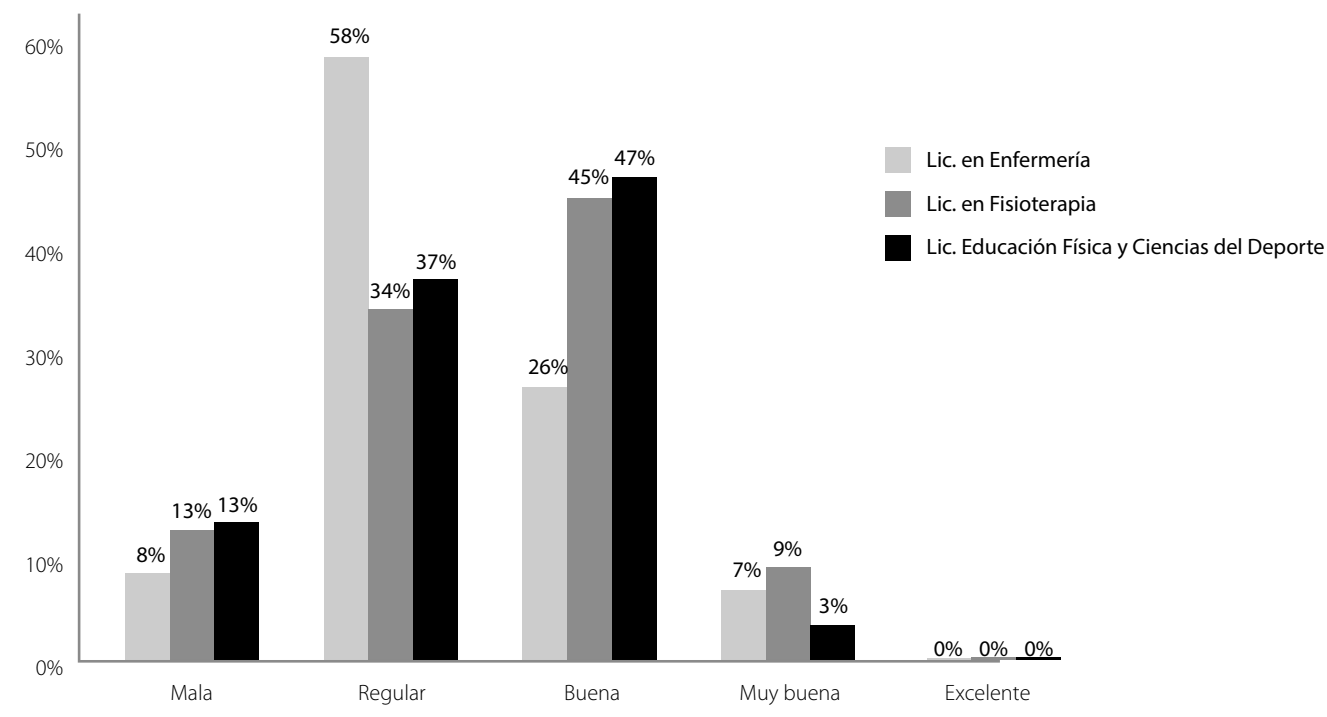

Fuente: Cuestionario de hábitos alimentarios de Castro, Bellido y Pertega, 2010.

\section{Bibliografía}

1. Organización Mundial de la Salud (OMS). Nutrición. 2015.

2. Cedillo-Ramírez L, Correa-López LE, Vela-Ruiz JM, Perez-Acuña LM, Loayza-Castro JA, Cabello-Vela
CS, et al. Estilos de Vida de Estudiantes Universitarios de Ciencias de la Salud. Rev la Fac Med Humana [Internet]. 2016;16(2):57-65. Available from: http://revistas.urp.edu.pe/index.php/RFMH/article/view/670

3. Jiménez Diez O, Ojeda López RN. Estudiantes uni- 
versitarios y el estilo de vida. Rev Iberoam Prod académica y gestión Educ. 2017;4(8):1-15.

4. Córdoba A, Carmona M, Terán O, Márquez O. Relación del estilo de vida y su estado de nutición en estudiantes universitarios: estudio descriptivo de corte transversal. 2013;

5. Antonella $R$, Vidal $P$, Brassesco B, Viola L, Aballay L. Estado nutricional en estudiantes universitarios: su relación con el número de ingestas alimentarias diarias y el consumo de macronutrientes. Nutr Hosp. 2015;31(4):1748-56.

6. Rizo B, González B, Cortés R. Calidad de la dieta y estilos de vida en estudiantes de Ciencias de la Salud. Nutr Hosp. 2014;1:153-7.

7. Becerra F, Vargas ME. Estado nutricional y consumo de alimentos de estudiantes universitarios admitidos a nutrición y dietética en la Universidad Nacional De Colombia. Rev Salud Pública. 2016;17(5):762-75.

8. Saliba TA, De Sandre AS, Saliba Moimaz SA, Saliba Garbin CA. Perfil de estilo de vida individual de estudiantes de primer año de odontología de la Universidad de Aracatuba, Brasil - 2015. Univ y Salud [Internet]. 2017;19(2):258. Available from: http:// revistas.udenar.edu.co/index.php/usalud/article/ view/3016

9. Chau C, Vilela P. Variables asociadas a la salud física y mental percibida en estudiantes universitarios de Lima. Lib Rev Peru Psicol [Internet]. 2017;23(1):89102. Available from: http://ojs3.revistaliberabit. com/index.php/Liberabit/article/view/51/51

10. Olivero N, Benites J, García A, Bello C. Estilos de vida en estudiantes de una universidad pública. Cátedra Villarreal. 2016;4:57-72.

11. Tamayo Caballero $C$, Alcocer $R \mathrm{H}$, Choque $\mathrm{S} L$, Chuquimia N A, Condori S P, Gutierrez F I, et al. Factores y determinantes del consumo de comida chatarra en estudiantes de la facultad de medicina, enfermería, nutrición y tecnología medica, La Paz-Bolivia 2016. Cuad Hosp Clínicas [Internet]. 2016;57(3). Available from: http://www.revistasbolivianas.org.bo/scielo. php?pid $=$ S1652-67762016000300005\&script $=$ sci arttext\&tlng $=$ es

12. Beltrán $M$, Gómez $C$. Estilo de vida en jóvenes de la facultad de Psicología de la Fundación Universitaria de los Libertadores. Los Lib [Internet]. 2016; Availa- ble from: http://repository.libertadores.edu.co/bitstream/1137/958/1/beltrancorredorMarily.pdf

13. Farfán $M$, Olarte $G$. Prevalencia de sobrepeso, obesidad, comportamientos alimentarios y actividad física en estudiantes universitarios en Boyacá. Rev Investig en Salud Univ Boyacá [Internet]. 2014;4:22835. Available from: http://doi.org/10.17843/rpmesp.2016.332.2170

14. Castro P, Bellido D, Pertega S. Elaboración de un cuestionario de hábitos alimentarios para pacientes con sobrepeso y obesidad. Endocrinol y Nutr. 2010;57(4):130-9.

15. Secretaria de Salud. Reglamento de la Ley General de Salud en Materia de Investigaciones para la Salud. [Internet]. Ley General de Salud. 1987. Available from: http://www.cofepris.gob.mx/MJ/Paginas/Reglamentos.aspx

16. Fernández $N$, Romero $M$, Huerta $G$, Barradas $M$. Ingesta de alimentos: ¿balanceada o desbalanceada? Rev Iberoam Prod académica y gestión Educ. 2015;1-9.

17. De Piero A, Bassett N, Rossi A, Samman N. Tendencia en el consumo de alimentos de estudiantes universitarios. Nutr Hosp. 2015;31(4):1824-31.

18. Durán $S$, Crovetto $M$, Espinoza $V$, Mena F, Oñate $G$ Fernández $M$, et al. Caracterización del estado nutricional, hábitos alimentarios y estilos de vida de estudiantes universitarios chilenos: estudio multicéntrico. Rev Med Chile. 2017;145:1405-13.

19. Chávez Valenzuela $M E$, Salazar $C$, Hoyos Ruíz G, Bautista Jacobo A, González Lomelí D, Ogarrio Perkins CE. Actividad física y enfermedades crónicas no transmisibles de estudiantes mexicanos en función del género. Retos [Internet]. 2018;33(1):169-74. Available from: http://eds.b.ebscohost.com/eds/ pdfviewer/pdfviewer?vid $=13 \&$ sid $=53635$ a 89 . $4 f c 4-41$ e5-8521-ebecf4c8152b\% 40pdc-v-sessmgr01

20. López Gutiérrez PP, Rejón Orantes J del C, Escobar Castillejos D, Roblero Ochoa SR, Dávila Esquivel MT, Mandujano Trujillo ZP. Conocimientos nutricionales en estudiantes universitarios del sector público del Estado de Chiapas, México. Investig en Educ Médica [Internet]. 2017;6(24):228-33. Available from: http://linkinghub.elsevier.com/retrieve/pii/ S2007505716300795 